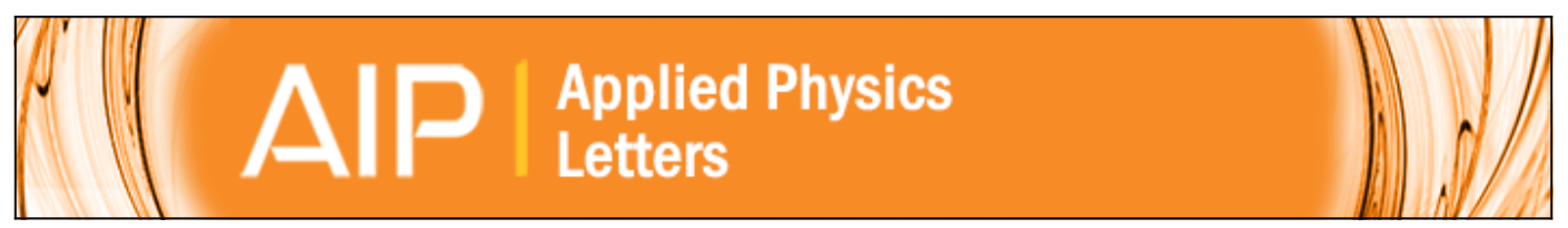

\title{
Systematic optimization of quantum junction colloidal quantum dot solar cells
}

Huan Liu, David Zhitomirsky, Sjoerd Hoogland, Jiang Tang, Illan J. Kramer, Zhijun Ning, and Edward H. Sargent

Citation: Applied Physics Letters 101, 151112 (2012); doi: 10.1063/1.4757866

View online: http://dx.doi.org/10.1063/1.4757866

View Table of Contents: http://scitation.aip.org/content/aip/journal/apl/101/15?ver=pdfcov

Published by the AIP Publishing

\section{Articles you may be interested in}

Enhanced performance of branched TiO2 nanorod based Mn-doped CdS and Mn-doped CdSe quantum dotsensitized solar cell

J. Appl. Phys. 117, 163104 (2015); 10.1063/1.4918913

Photocurrent extraction efficiency in colloidal quantum dot photovoltaics

Appl. Phys. Lett. 103, 211101 (2013); 10.1063/1.4831982

Organic/inorganic hybrid pn-junction between copper phthalocyanine and CdSe quantum dot layers as solar cells J. Appl. Phys. 112, 044507 (2012); 10.1063/1.4747835

Increase in photocurrent by optical transitions via intermediate quantum states in direct-doped InAs/GaNAs strain-compensated quantum dot solar cell

J. Appl. Phys. 109, 024301 (2011); 10.1063/1.3533423

Nanowire-quantum-dot solar cells and the influence of nanowire length on the charge collection efficiency

Appl. Phys. Lett. 95, 193103 (2009); 10.1063/1.3258490

\section{AIP $\left.\right|_{\text {APL Photonics }}$}

APL Photonics is pleased to announce Benjamin Eggleton as its Editor-in-Chief

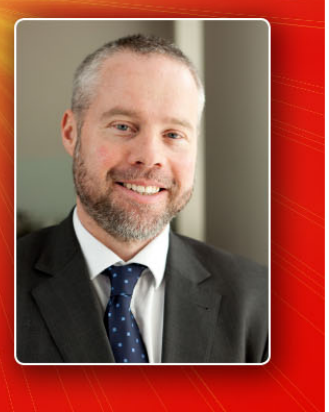




\title{
Systematic optimization of quantum junction colloidal quantum dot solar cells
}

\author{
Huan Liu, ${ }^{1}$ David Zhitomirsky, ${ }^{2}$ Sjoerd Hoogland, ${ }^{2}$ Jiang Tang, ${ }^{3}$ Illan J. Kramer, ${ }^{2}$ \\ Zhijun Ning, ${ }^{2}$ and Edward $\mathrm{H}$. Sargent ${ }^{2, a)}$ \\ ${ }^{1}$ School of Optical and Electronic Information, Huazhong University of Science and Technology, \\ 1037 Luoyu Rd., Wuhan, Hubei 430074, China \\ ${ }^{2}$ Department of Electrical and Computer Engineering, University of Toronto, 10 King's College Road, \\ Toronto, Ontario M5S 3G4, Canada \\ ${ }^{3}$ Wuhan National Laboratory for Optoelectronics, Huazhong University of Science and Technology, \\ 1037 Luoyu Rd., Wuhan, Hubei 430074, China
}

(Received 24 July 2012; accepted 21 September 2012; published online 10 October 2012)

\begin{abstract}
The recently reported quantum junction architecture represents a promising approach to building a rectifying photovoltaic device that employs colloidal quantum dot layers on each side of the $\mathrm{p}-\mathrm{n}$ junction. Here, we report an optimized quantum junction solar cell that leverages an improved aluminum zinc oxide electrode for a stable contact to the n-side of the quantum junction and silver doping of the p-layer that greatly enhances the photocurrent by expanding the depletion region in the n-side of the device. These improvements result in greater stability and a power conversion efficiency of $6.1 \%$ under AM1.5 simulated solar illumination. (C) 2012 American Institute of Physics. [http://dx.doi.org/10.1063/1.4757866]
\end{abstract}

Colloidal quantum dots (CQDs) are attractive materials for inexpensive, room temperature and solution processed optoelectronics. These materials have been employed in light emitting devices, ${ }^{1}$ field effect transistors (FETs), ${ }^{2,3}$ and photovoltaics. $^{4-7}$

In photovoltaics, CQD films are typically combined with a wide-bandgap bulk semiconductor which allows for the formation of a $p-n$ junction. This limits the benefits of bandgap tuning in CQD films, since redesign of the bulk semiconductor is required to maintain performance due to band alignment. ${ }^{8}$ The quantum junction (QJ) architecture ${ }^{9}$ has obviated the need for bulk semiconductor materials, and has enabled an efficient implementation of a $p-n$ junction where both sides of the junction were capable of being quantum-tuned. This has opened a path towards all-room temperature processing from solution of the entire junction, obviating the need for the high-temperature sintering steps required when working with many relevant metal-oxide electrodes.

The QJ presents an attractive approach to building junctions of varying quantum dot sizes and thus bandgaps. To fulfil its potential in single-junction devices, the materials and the architecture need to be optimized for a bandgap range centered at $1.3 \mathrm{eV} .{ }^{10}$ We took the view that, through careful engineering of electrode materials, and by tuning the CQD properties, we could achieve greater power conversion efficiencies. Prior reports of QJ devices have relied on an indium tin oxide (ITO) transparent contact; an overlying, highly doped PbS CQD p-layer; a lightly doped, PbS CQD n-layer; the latter contacted using a final Al ohmic metal contact. $^{9}$

CQD films typically have low diffusion lengths, in the range from $10-100 \mathrm{~nm} .{ }^{11,12}$ As a result, extending the deple-

\footnotetext{
${ }^{\text {a) }}$ Author to whom correspondence should be addressed. Electronic mail: ted.sargent@utoronto.ca.
}

tion region as far as possible into a lightly doped n-layer is expected to enhance charge collection and thereby increase the photocurrent. This can be achieved if the doping in the p-layer can be considerably increased.

With this goal in mind, we investigated related material systems in which dopants have been introduced during synthesis. Ag ions have been used in doping InAs CQDs. ${ }^{13}$ The valency of $\mathrm{Ag}$ is suited to p-doping of PbS CQDs as well, providing one extra hole for each $\mathrm{Pb}$ atom that is replaced by $\mathrm{Ag}$ in the $\mathrm{PbS}$ lattice.

We therefore explored the addition of $\mathrm{Ag}$ during $\mathrm{PbS}$ CQDs synthesis. PbS CQDs of various sizes are fabricated following a modified published recipe. ${ }^{14,15}$ For Ag incorporation, the $\mathrm{Ag}$ precursor solution was prepared by dissolving $144 \mathrm{mg}$ of $\mathrm{AgCl}$ in $10 \mathrm{ml}$ of oleylamine (OLA). After the injection of bis(trimethylsilyl) sul-fide (TMS) for the synthesis of CQDs, the heating mantle was turned off to let the flask temperature decrease to $\sim 75^{\circ} \mathrm{C}$. $1.5 \mathrm{ml}$ of the $\mathrm{Ag}$ precursor and $3 \mathrm{ml}$ of OLA were then injected and the flask temperature was maintained at $75^{\circ} \mathrm{C}$ for $30 \mathrm{~min}$, after which it was allowed to cool to $36^{\circ} \mathrm{C}$. The procedure results in CQDs having $\mathrm{Ag}$ impurities incorporated, as verified using X-ray photoelectron spectroscopy (XPS) (S1). ${ }^{21}$ Doping densities were obtained using FET measurements, employing a $\mathrm{SiO}_{2} / \mathrm{Si}$ architecture with $\mathrm{Au}$ drain and source contacts, where a $40 \mathrm{~nm}$ film of CQDs treated with tetramethyl ammonium hydroxide in methanol formed the channel. The extracted doping densities (Figure 1(a) inset) confirmed that the doping in the p-layer had increased substantially as a result of the Ag incorporation. The Ag doping approach resulted in doping densities approaching $10^{19} \mathrm{~cm}^{-3}$ found to be optimal for device performance.

We then fabricated p-n devices using a layer-by-layer approach, ${ }^{9}$ with a $300 \mathrm{~nm}$ n-layer processed in an inert environment with tetrabutylammonium iodide ligands in methanol $(10 \mathrm{mg} / \mathrm{ml})$ on a $50 \mathrm{~nm}$ air ambient processed p-layer, with tetramethyl ammonium hydroxide in methanol $(10 \mathrm{mg} / \mathrm{ml})$ as 

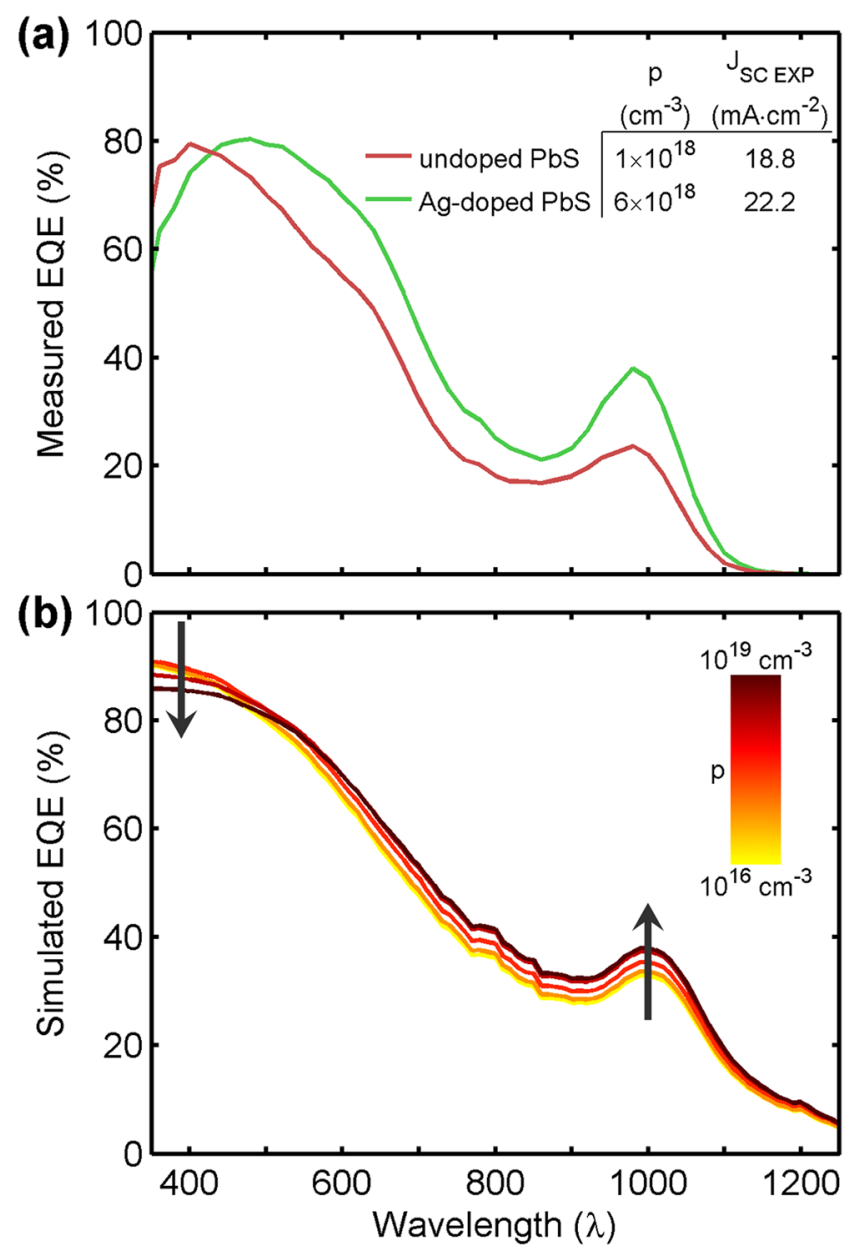

FIG. 1. (a) EQE of devices employing undoped and Ag-doped CQDs showing that Ag doping leads to a greater depletion in the n-layer, inset: hole density (p) as measured by FETs for films made with undoped and Ag-doped $\mathrm{PbS}$ CQDs; expected $\mathrm{J}_{\mathrm{SC}}$ shown as calculated from (a). (b) Simulated EQE curves showing an enhancement in the near infrared and across part of the visible, while a slight reduction around $400 \mathrm{~nm}$ due to decreased depletion in the p-layer.

the ligand. A thin, highly doped p-layer, and a thicker, lightly doped n-layer ensure high absorption and depletion in the nlayer, excellent for charge generation and collection. External quantum efficiency (EQE) measurements revealed the benefit from increased doping in the p-layer as a result of Ag incorpo- ration. The incident spectrum for EQE measurements was generated by passing the output of a $400 \mathrm{~W}$ Xe lamp through a monochromator and using appropriate order-sorting filters. The resultant monochromatic beam also passed through an optical chopper operating at $220 \mathrm{~Hz}$ coupled to the input of a lock-in amplifier. The EQE (Figure 1(a)) was greatly enhanced across most of the visible spectrum, and markedly so at the infrared excitonic peak where absorption occurs deeper into the device compared to the case of visible-light absorption. The EQE showed a slight decrease at shorter wavelengths, consistent with a decrease in the depletion depth inside the front-side p-layer due to its increased doping.

To explore further this picture of doping and its impact on depletion in the quantum junction, we constructed an optoelectronic model using previously established approaches. ${ }^{16-18}$ The resulting EQE curves (Figure 1(b)) demonstrated that, as the doping in the p-layer is increased, the EQE improves across most of the visible and infrared spectrum. Meanwhile, the EQE is reduced at high-energy wavelengths, a fact attributable to the decrease in the depletion region on that side of the junction. The EQE curves further suggest that the overall improvement in EQE across the entire spectrum outweighs any reduction in EQE due to less depletion in the p-layer, also consistent with experimental results.

For a device to be attractive for photovoltaics, it would also need to have excellent long term stability and temperature resilience. The presently employed $\mathrm{Al}$ contact is known to react with halides ${ }^{19}$ that are used during the fabrication of the n-type film. A highly doped n-type aluminum zinc oxide (AZO) layer could therefore potentially serve to enhance stability while improving current collection by blocking hole recombination (Figure 2(a)). AZO is readily sputtered at room temperature, ${ }^{20,21}$ and a $30 \mathrm{~nm}$ contact was deposited at a rate of $0.4 \AA / \mathrm{s}$ by sputtering, followed by thermal evaporation of silver $(200 \mathrm{~nm}$ thick) at a rate of $1.5 \AA / \mathrm{s}$ with base pressure of $1 \times 10^{-7}$ mbar. Figure 2(b) shows the temporal stability of our AZO devices. These devices were encapsulated in a glovebox and tested in an air ambient environment. Encapsulation was done by sealing the device in an epoxy (NOA88, Norland Products Inc), and then curing with an $8 \mathrm{~W}$ UV lamp (UVLMS38, UVP Inc.) for $30 \mathrm{~min}$. The devices retained performance for over $60 \mathrm{~h}$ under continuous (a)

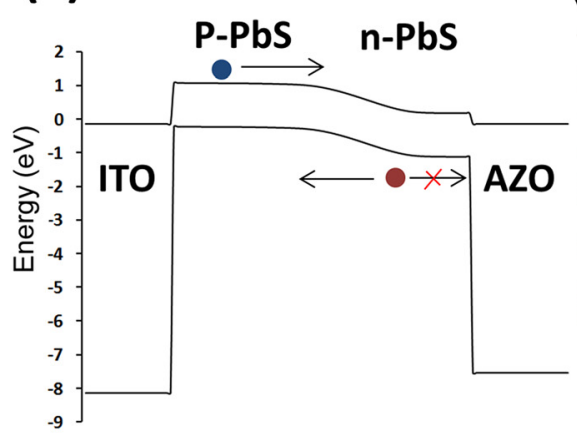

(b)

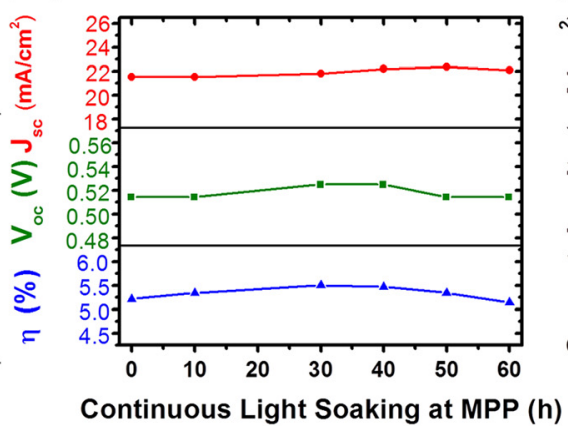

(c)

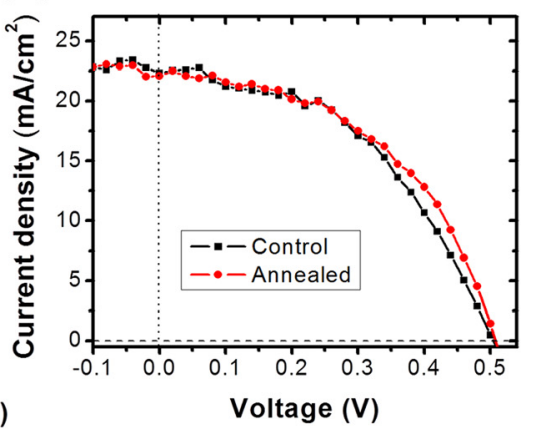

FIG. 2. (a) Energy band diagrams of device employing an AZO electrode in place of an Al contact. X-axis is not to scale. The AZO also serves as a hole blocking layer. (b) Stability of a $>5 \%$ devices across all performance metrics for $60 \mathrm{~h}$ (c) Temperature stability of a QJ device after annealing at $90{ }^{\circ} \mathrm{C}$ for $3 \mathrm{~h}$, showing little change in JV characteristics. 
illumination. The same devices also exhibited temperature stability to $90^{\circ} \mathrm{C}$ (Figure 2(c)), highlighting the robustness of the AZO approach.

Current-voltage characteristics were measured using a Keithley 2400 source-meter in $\mathrm{N}_{2}$ ambient. The solar spectrum at AM1.5 was simulated with a Xe lamp and filters (Solar Light Company Inc.) with measured intensity at $100 \mathrm{~mW}$ $\mathrm{cm}^{-2}$. Combining the silver doping and AZO contact optimizations, we were able to improve power conversion efficiency, principally by collecting more photocurrent. These advances resulted in devices exhibiting a PCE of $6.1 \%$. Figure 3(a) shows the J-V curve of a champion device, with an open circuit voltage of $0.52 \mathrm{~V}$, short circuit current $\left(\mathrm{J}_{\mathrm{SC}}\right)$ of $23.3 \mathrm{~mA} / \mathrm{cm}^{2}$, and fill factor (FF) of $50 \%$; where most of the improvement is reflected in the $\mathrm{J}_{\mathrm{SC}}$ and FF compared with previous reports. ${ }^{9}$ This is consistent with an enhanced depletion region and thus more efficient carrier collection, furthermore aided by hole blocking afforded by using an AZO contact. We constructed sixty QJ devices (Fig. 3(b)) over the course of one month using different batches of PbS CQDs to test the reproducibility of fabrication approach. The average efficiency was $5.6 \%$ and the standard deviation was $0.2 \%$. Such a high reproducibility compares favourably with performance variation in previously reported depleted heterojunction devices. $^{6}$

We have shown that by engineering the CQD material through doping and by appropriately choosing ohmic electrodes to our CQD films, we are able to collect more current

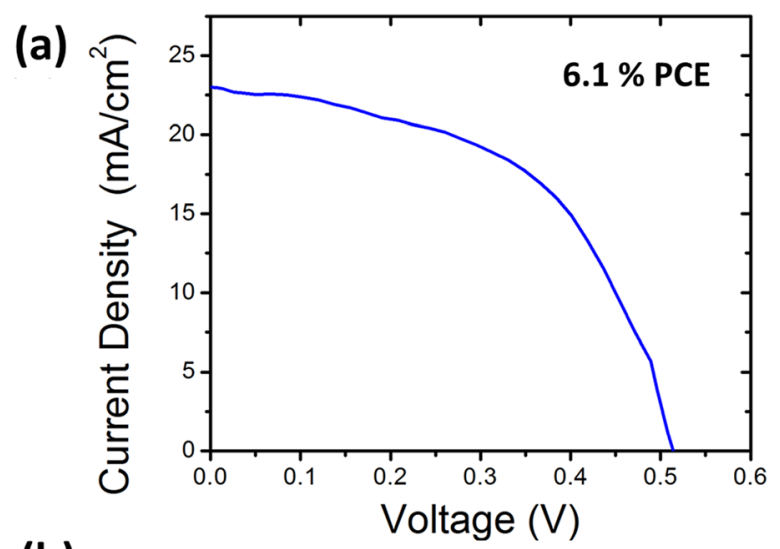

(b)

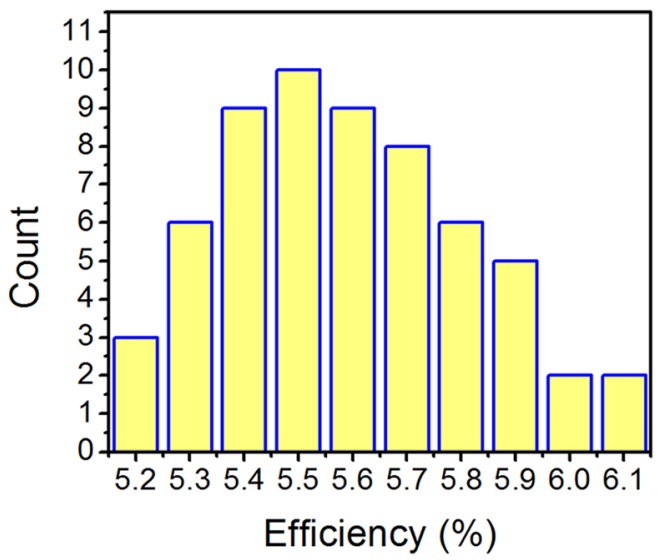

FIG. 3. (a) J-V curve of best performing device showing a PCE of $6.1 \%$. (b) Performance of 60 devices made over 1 month, showing high reproducibility and little deviation in PCE $(\mathrm{PCE}=5.6 \pm 0.2 \%)$. and increase efficiency. Furthermore, the choice of the ohmic contact can have a drastic impact on device stability by limiting unwanted chemical reactions and forming a favourable energy alignment for further enhancement in charge collection. We achieve highly reproducible, high performance devices with excellent temperature and temporal stability. This work opens a path towards further optimization of related QJ devices by appropriately tuning the doping and choice of electrical contacts to guarantee efficient charge collection and high efficiency.

We thank Angstrom Engineering and Innovative Technology for useful discussions regarding material deposition methods and control of glovebox environment, respectively. The authors would like to acknowledge the technical assistance and scientific guidance of E. Palmiano, R. Wolowiec, and D. Kopilovic. This publication is based on part of work supported by Award KUS-11-009-21, made by King Abdullah University of Science and Technology (KAUST), by the Ontario Research Fund Research Excellence Program, and by the Natural Sciences and Engineering Research Council (NSERC) of Canada. D. Zhitomirsky acknowledges the financial support through the NSERC CGS D Scholarship.

${ }^{1}$ L. Sun, J. J. Choi, D. Stachnik, A. C. Bartnik, B.-R. Hyun, G. G. Malliaras, T. Hanrath, and F. W. Wise, Nat. Nanotechnol. 7, 369 (2012).

${ }^{2}$ T. P. Osedach, N. Zhao, T. L. Andrew, P. R. Brown, D. D. Wanger, D. B. Strasfeld, L.-Y. Chang, M. G. Bawendi, and V. Bulović, ACS Nano 6, 3121 (2012).

${ }^{3}$ D. V. Talapin and C. B. Murray, Science 310, 86 (2005).

${ }^{4}$ A. G. Pattantyus-Abraham, I. J. Kramer, A. R. Barkhouse, X. Wang, G. Konstantatos, R. Debnath, L. Levina, I. Raabe, M. K. Nazeeruddin, M. Gratzel, and E. H. Sargent, ACS Nano 4, 3374 (2010).

${ }^{5}$ L. Etgar, W. Zhang, S. Gabriel, S. G. Hickey, M. K. Nazeeruddin, A. Eychmüller, B. Liu, and M. Grätzel, Adv. Mater. 24, 2202 (2012).

${ }^{6}$ J. Tang, K. W. Kemp, S. Hoogland, K. S. Jeong, H. Liu, L. Levina, M. Furukawa, X. Wang, R. Debnath, D. Cha, K. W. Chou, A. Fischer, A. Amassian, J. B. Asbury, and E. H. Sargent, Nature Mater. 10, 765 (2011).

${ }^{7}$ B.-R. Hyun, Y.-W. Zhong, A. C. Bartnik, L. Sun, H. D. Abruña, F. W. Wise, J. D. Goodreau, J. R. Matthews, T. M. Leslie, and N. F. Borrelli, ACS Nano 2, 2206 (2008).

${ }^{8}$ H. Liu, J. Tang, I. J. Kramer, R. Debnath, G. I. Koleilat, X. Wang, A. Fisher, R. Li, L. Brzozowski, L. Levina, and E. H. Sargent, Adv. Mater. 23, 3832 (2011).

${ }^{9}$ J. Tang, H. Liu, D. Zhitomirsky, S. Hoogland, X. Wang, M. Furukawa, L. Levina, and E. H. Sargent, Nano Lett. 12, 4889 (2012).

${ }^{10}$ C. H. Henry, J. Appl. Phys. 51, 4494 (1980).

${ }^{11}$ J. M. Luther, M. Law, M. C. Beard, Q. Song, M. O. Reese, R. J. Ellingson, and A. J. Nozik, Nano Lett. 8, 3488 (2008).

${ }^{12}$ K. W. Johnston, A. G. Pattantyus-Abraham, J. P. Clifford, S. H. Myrskog, S. Hoogland, H. Shukla, E. J. D. Klem, L. Levina, and E. H. Sargent, Appl. Phys. Lett. 92, 122111 (2008).

${ }^{13}$ D. Mocatta, G. Cohen, J. Schattner, O. Millo, E. Rabani, and U. Banin, Science 332, 77 (2011).

${ }^{14}$ M. A. Hines and G. D. Scholes, Adv. Mater. 15, 1844 (2003).

${ }^{15}$ J. Tang, L. Brzozowski, D. A. R. Barkhouse, X. Wang, R. Debnath, R. Wolowiec, E. Palmiano, L. Levina, A. G. Pattantyus-Abraham, D. Jamakosmanovic, and E. H. Sargent, ACS Nano 4, 869 (2010).

${ }^{16}$ D. Zhitomirsky, I. J. Kramer, A. J. Labelle, A. Fischer, R. Debnath, J. Pan, O. M. Bakr, and E. H. Sargent, Nano Lett. 12, 1007 (2012).

${ }^{17}$ I. J. Kramer, L. Levina, R. Debnath, D. Zhitomirsky, and E. H. Sargent, Nano Lett. 11, 3701 (2011).

${ }^{18}$ I. J. Kramer and E. H. Sargent, ACS Nano 5, 8506 (2011).

${ }^{19}$ J. G. Chen, T. P. Beebe, J. E. Crowell, and J. T. Yates, J. Am. Chem. Soc. 109, 1726 (1987).

${ }^{20}$ G. I. Koleilat, X. Wang, A. J. Labelle, A. H. Ip, G. H. Carey, A. Fischer, L. Levina, L. Brzozowski, and E. H. Sargent, Nano Lett. 11, 5173 (2011).

${ }^{21}$ See supplementary material at http://dx.doi.org/10.1063/1.4757866 for XPS spectra and composition analysis of Ag-doped PbS CQDs. 